\title{
Mortalidad Materna y Perinatal en los Estados Hipertensivos del Embarazo
}

\author{
Dres.: Alvaro Velasco $\mathrm{Ch}^{*}$ y Hilma Barriga G.**
}

\section{AGRADECIMIENTO}

Los autores agradecen la colaboración de Profesores, Adjuntos, Residentes y Personal paramédico del Departamento de Ginecología y Obstetricia que han colaborado activamente en la ejecución del presente trabajo.

Al Dr. Armando Rojas P. por el trabajo estadístico computarizado.

\section{ANALISIS COMPARATIVO DE DOS TIPOS DE TRATAMIENTO}

En junio de 1981 se inició una nueva rutina de tratamiento para las enfermedades hipertensivas del embarazo, hasta entonces delineado a base de diuréticos, fenobarbital e hipotensores, solamente aplicados an caso de hipertensión crónica. Las conductas en la mayoría de los casos con un criterio conservatorio, estaban dirigidas a prolongar la gestación para lograr una mayor maduración fetal con el objeto de disminuir la mortalidad perinatal, aún después de los accidentes eclámpticos.

\footnotetext{
* Profesor Asociado. Departamento de Ginecología y Obstetricia.

* * Residente III. Departamento de Ginecologia y Obstetricia.
}

A partir de la fecha mencionada se inicia un tratamiento a base de sustancias vasodilatadoras e hipotensoras, con interrupción sistemática del embarazo cuando las condiciones maternas y fetales, demostradas por monitoría, se encontraban fuertemente comprometidas. Se utilizó con mayor frecuencia, la cesárea como método para interrumpir el embarazo y se suspendieron los diuréticos y barbitúricos formulados por períodos prolongados.

Los trabajos de Campbell y McGillivray, 1975 (1), y de McGillivray (2) entre otros autores, manifiestan que los tiacídicos son ineficaces con fines profilácticos y terapéuticos $y$ tienen efectos secundarios nocivos sobre el recién nacido.

Los barbitúricos aplicados durante períodos prolongados han sido cuestionados por su acción depresora sobre la función muscular respiratoria del feto in útero y por su acción depresora sobre la maduración pulmonar, factores que inciden en un aumento de los problemas de dificultad del recién nacido.

El presente trabajo tiene además la intención de demostrar ampliamente la mortalidad perinatal, estudiándola en todos los grupos de la clasificación de los estados hipertensivos, pues solamente se han presentado observaciones muy limi- 
tadas relacionadas especialmente con la mortalidad en la eclampsia, pero no con los estados de preeclampsia ni con estados de enfermedad hipertensiva crónica, Sánchez Torres, $(3,4)$, Rojas L. (5), Rodríguez S. (6).

\section{MATERIAL Y METODOS}

Para el estudio comparativo entre los dos tipos de tratamiento se analizaron dos grupos de pacientes hipertensas, hospitalizadas en el Departamento de Toxemias del Instituto Materno Infantil de Bogotá, as í:

\section{Grupo A}

A este grupo pertenecen las pacientes hospitalizadas en el período comprendido desde julio 2 de 1980 hasta julio 2 de 1981, que fueron tratadas con las normas existentes en el servicio en dicha fecha, que comprendía uso diario de diuréticos, sedantes tipo fenobarbital durante tiempo prolongado; excepcionalmente se utilizaron vasodilatadores e hipotensores, los cuales se empleaban casi exclusivamente en pacientes con hipertensión crónica; la posición en decúbito era la adoptada espontáneamente por la paciente; se sometían rutinariamente a dieta hiposalina. En caso de eclampsia se trataron con sulfato de magnesia y fenobarbital y raramente se aplicaron vasodilatadores por vía parenteral.

La conducta obstétrica era en la mayoría de los casos espectante, con el objeto de lograr una mayor maduración fetal. El servicio no disponía de ecógrafo ni monitor fetal.

\section{Grupo B}

Estas pacientes fueron tratadas durante el período comprendido desde julio 2 de 1981 hasta julio 2 de 1982, con un nuevo esquema consistente en reposo en decúbito lateral, vasodilatadores e hipotensores, utilizados por vía oral o paren- teral. No se administraron diuréticos, dieta hiposalina ni fenobarbital. En la eclampsia se administraron sulfato de magnesia y vasodilatadores por vía parenteral. Para este período se dispuso de ecógrafo y monitor para la evaluación fetal antenatal y la conducta obstétrica fue más activa, efectuando la interrupción del embarazo más tempranamente, según las condiciones fetales.

\section{Población analizada}

\section{Grupo A}

Durante este período se atendieron en el Instituto 13.776 partos y se obtuvieron 13.488 nacidos vivos y 356 nacidos muertos. 363 pacientes presentaron enfermedad hipertensiva $(2.68 \%)$.

\section{Grupo B}

En este período se atendieron 10.395 partos y se obtuvieron 10.139 nacidos vivos y 343 nacidos muertos; se encontraron 391 pacientes hipertensas $(3.76 \%)$. El segundo período coincide con la época en la cual el hospital ha disminuido el ingreso de pacientes de bajo riesgo (envíadas a otro hospital) lo que ocasiona el ligero aumento en el porcentaje de pacientes hipertensas.

No fue posible obtener el dato de mortalidad neonatal durante estos períodos. El análisis de la mortalidad materna se obtuvo por la revisión de las historias de la Sección de Toxemias y de la estadística general del Instituto Materno Infantil.

\section{ANALISIS DE RESULTADOS}

Las pacientes fueron analizadas según la siguiente clasificación:

I Hipertensión inducida por el embarazo (H.I.E.) subdividida en a) preeclampsia leve y moderada (P.E.), pacientes con hipertensión menor de $170 / 120$. b) pacientes con preeclamp- 
sia grave o inminencia de eclampsia (I.E.), aquellas con tensión arterial igual o mayor de $170 / 120$ y con síntomas de cefálea intensa, epigastralgia y visión borrosa. c). Eclampsia (E).

II Enfermedad vascular hipertensiva crónica (E.V.H.C.) a) E.V.H.C. sola b) E.V.C.H. con H.I.E. sobreagregada, la cual se divide a su vez en EVHC con preeclampsia leve o moderada sobreagregada, EVHC con inminencia de eclampsia sobreagregada y EVHC con eclampsia sobreagregada.

\section{Grupo $A$}

En este grupo se trataron 363 pacientes hipertensas con los siguientes diagnósticos: H.I.E.

Preeclampsia: 116 pacientes $(31.96 \%)$, inminencia de eclampsia 78 pacientes $(21,49 \%)$, eclampsia 62 pacientes (17. $08 \%$.

Cuadro No. 1

DIAGNOSTICO DE EGRESO VS. TRATAMIENTO

\begin{tabular}{|c|c|c|c|}
\hline & GRUPO A & GRUPO & $B$ \\
\hline Sin dato & 0.28 & 0.00 & \\
\hline Preeclampsia & $\begin{array}{r}116 \\
31.96\end{array}$ & $\begin{array}{r}122 \\
31.20\end{array}$ & $x^{2}=0.04$ \\
\hline Inm. de Eclampsia & $\begin{array}{r}78 \\
21.49\end{array}$ & $\begin{array}{r}54 \\
13.81\end{array}$ & $x^{2}=7.68$ \\
\hline Eclampsia & $\begin{array}{r}62 \\
17.08\end{array}$ & $\begin{array}{r}76 \\
19.44\end{array}$ & $x^{2}=0.69$ \\
\hline EVHC + Preeciampsia & $\begin{array}{r}26 \\
7.16\end{array}$ & $\begin{array}{r}60 \\
15.35\end{array}$ & $x^{2}=12.47$ \\
\hline EVHC + Inm.EClampsia & $\begin{array}{r}47 \\
12.95\end{array}$ & $\begin{array}{r}44 \\
11.25\end{array}$ & $x^{2}=0.50$ \\
\hline EVHC + Eclampsia & $\begin{array}{r}14 \\
3.86\end{array}$ & $\begin{array}{r}20 \\
5.12\end{array}$ & $\ddot{x}^{2}=0.69$ \\
\hline EVHC & $\begin{array}{r}i 7 \\
4.68\end{array}$ & 2.81 & $x^{2}=i .84$ \\
\hline Otro & $\begin{array}{r}2 \\
0.55\end{array}$ & $\begin{array}{r}4 \\
1.02\end{array}$ & \\
\hline TOTAL & 363 & 391 & \\
\hline
\end{tabular}




\section{EVHC}

E.V.H.C. con preeclampsia sobreagregada 26 pacientes $(7.16 \%)$. E.V.H.C. con inminencia de eclampsia sobreagregada 47 pacientes $(12.95 \%)$, E.V. H.C. con eclampsia sobreagregada 14 pacientes $(3.86 \%)$, E.V.H.C. sola 17 pacientes $(4.68 \%)$. Ver cuadro No. 1 .

\section{Grupo B}

En este grupo se trataron 391 pacientes con los siguientes diagnósticos:

\section{H.I.E.}

Preeclampsia 122 pacientes $(31.20 \%)$, inminencia de eclampsia 54 pacientes $(13.81 \%)$, eclampsia 76 pacientes $(19.44 \%)$.

\section{E.V.H.C.}

E.V.H.C. con preeclampsia sobreagregada 60 pacientes $(15.35 \%)$, E.V. H.C. con inminencia de eclampsia sobreagregada 44 pacientes $(11.25 \%)$, E.V. H.C. con eclampsia sobreagregada 20 pacientes $(5.12 \%)$, E.V.H.C. sola, 11 pacientes $(2.81 \%)$. Ver cuadro No. 2 .
No hay diferencias significativas en los grupos analizados salvo en las pacientes de H.I.E. con inminencia de eclampsia $\left(X^{2}=7.68, p=0,01\right)$ y en EVHC con preeclampsia sobreagregada $\left(X^{2}=12.47\right.$, $\mathrm{p}=0.01$ ).

\section{MORTALIDAD MATERNA}

En el grupo A fallecieron 23 pacientes de las cuales 19 fallecieron por complicaciones propias de la toxemia y 4 por otras complicaciones; el índice depurado de mortalidad en la toxemia es del $5.23 \%$

Considerando que estas pacientes fueron eclámpticas, el índice de mortalidad en la eclampsia es del 25\% (19 pacientes fallecidas entre 76 pacientes eclámpticas).

En el grupo $A$ se atendieron 13.776 partos. La mortalidad por toxemia en relación al número de partos es de $137 x$ 100.000 .

En este período fallecieron en el Instituto Materno Infantil 46 pacientes, 19 por toxemia, lo que representa el $41 \%$.

Cuadro No. 2

TRATAMIENTO VS. CAUSAS DE MUERTE

\begin{tabular}{|rrrrrr|}
\hline & $\begin{array}{c}\text { NO HLBO } \\
\text { MUERTE }\end{array}$ & ACV & $\begin{array}{r}\text { ELENA } \\
\text { PULMONAR }\end{array}$ & SEPSIS & OTRA \\
\hline GRUPO A & 340 & 17 & 2 & 2 & 2 \\
& 93.66 & 4.68 & 0.55 & 0.55 & 0.55 \\
\hline \multirow{2}{*}{ GRUPO B } & 389 & 1 & 0 & 0 & 0 \\
& 51.66 & 0.13 & 0.00 & 0.00 & 0.00 \\
\hline
\end{tabular}




\section{GRUPOS DE ESTUDIO}

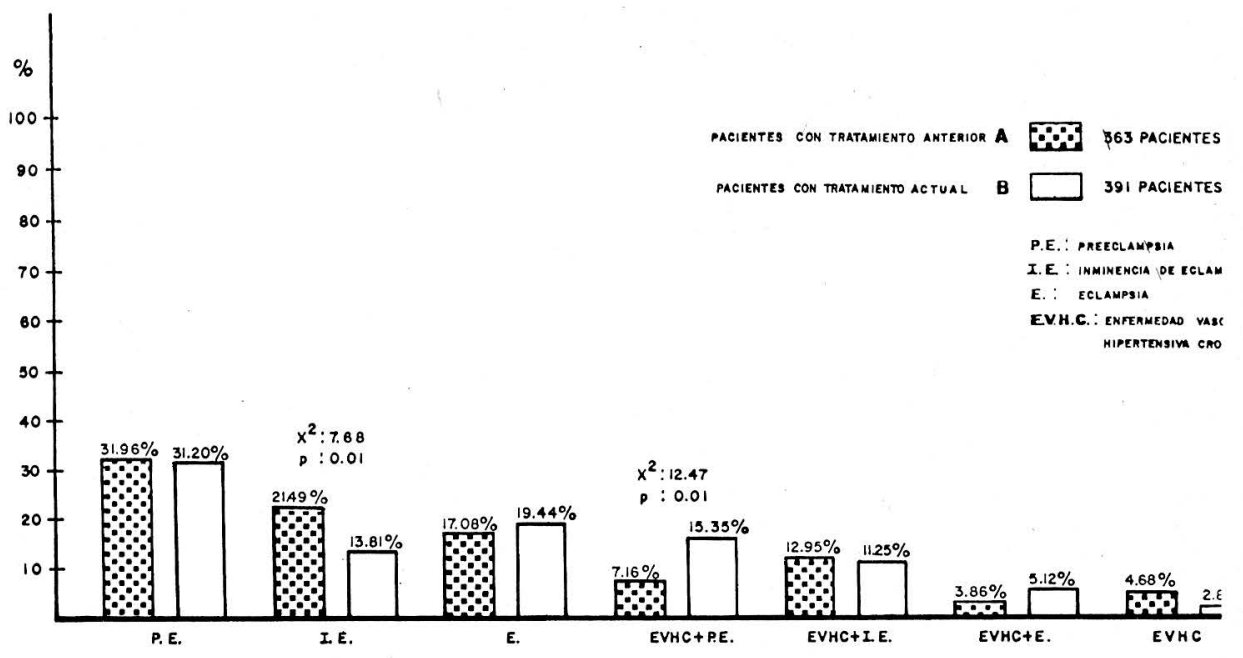

En el grupo B falleció una paciente por ACV que corresponde a una mortalidad materna del $0.26 \%$ en relación con las pacientes hipertensas. Esta paciente fue una eclámptica y durante este período se presentaron 96 pacientes eclámpticas, lc que representa una mortalidad del $1.04 \%$.

Ein el segundo período arializado (B) se atendieron 10.395 partos. La mortalidad por toxemia en relación al número de partos es de $9.6 \times 100.000$.

En este período fallecieron 23 pacientes, 1 por toxemia lo que representa el $4.34 \%$.

\section{CAUSAS DE MORTALIDAD}

En el grupo A se hallarcn 19 pacientes que fallecieron por causa directa de la toxemia: ACV en 17 pacientes y edema pulmonar en 2 pacientes.

En el grupo B fallece una paciente por ACV. (Ver cuadro No. 2). El accidente cerebro vascular es la causa predominante de la muerte en las pacientes toxémicas.

El $60 \%$ de pacientes del grupo $A$ estuvieron hospitalizadas en el Departamento de Toxemias y las otras fallecieron poco tiempo después de ingresadas, en el servicio de admision, sala de parto o sala de cuidados intensivos.

\section{MORBILIDAD MATERNA}

Las complicaciones detalladas en el cuadro 3 destacan en primer lugar el ACV como principal complicación de la hipertensión. En el grupo A se hallaron 18 pacientes de las cuales fallecen 17.

En el grupo $\mathrm{B}$, se encontraron 3 casos con ACV de las cuales fallece solamente una paciente, la diferencia es significativa ( $p-0.001)$. El mejor pronóstico de esta grave enfermedad puede ser debida al uso sistemático de vasodilatadores parenterales. 


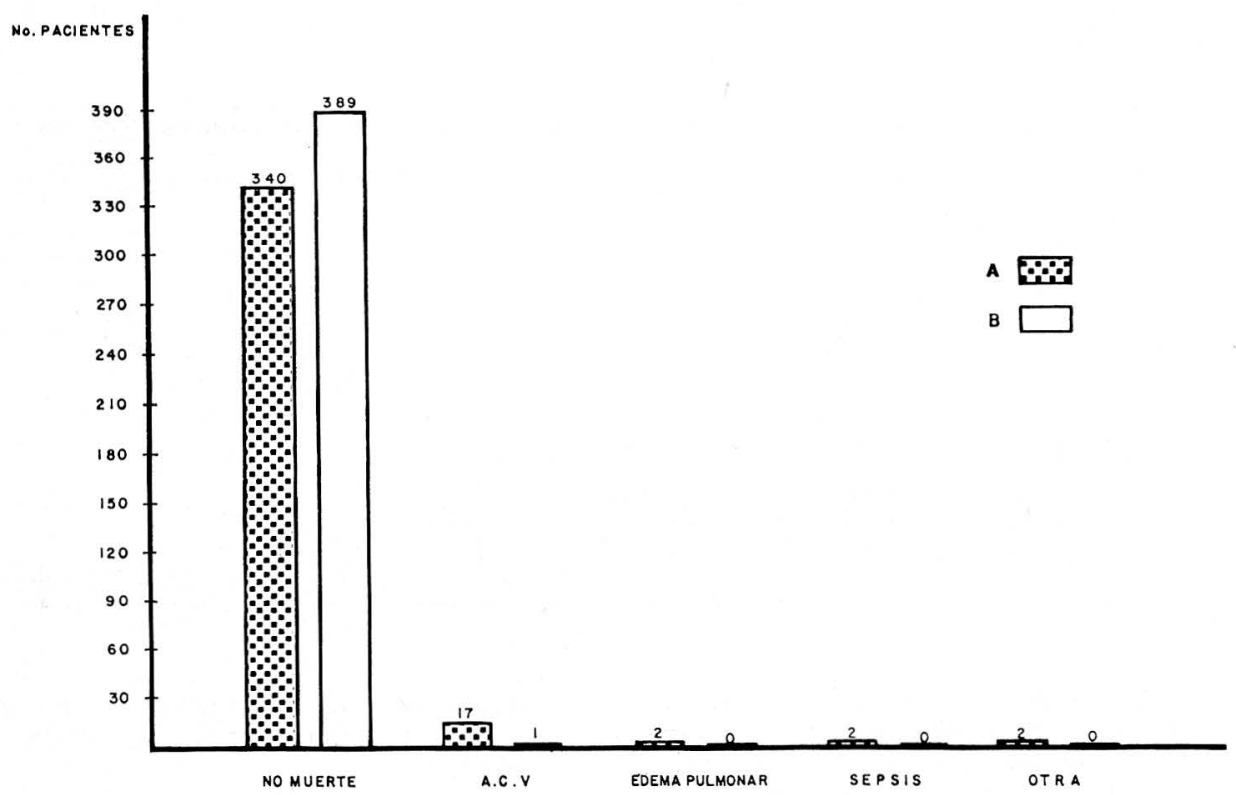

La hemorragia postparto se presentó en 9 pacientes del grupo A y 26 del grupo $B(p=0.01)$ no hay explicación aparente de esta situación.
No hubo diferencia significativa en el número de pacientes con IRA como tampoco lo hubo en las otras complicaciones maternas.

Cuadro No. 3

TRATAMIENTO VS. COMPLICACIONES MATERNAS

\begin{tabular}{|rrrrrrrrr|}
\hline & $\begin{array}{r}\text { SIN } \\
\text { DATO }\end{array}$ & $\begin{array}{c}\text { SIN COMPLI } \\
\text { CACIONES }\end{array}$ & ACV & AMNIONITIS & CID & $\begin{array}{r}\text { HEMORRA } \\
\text { GIA }\end{array}$ & IRA & $\begin{array}{c}\text { OTRAS } \\
\text { COMPLICACIONES }\end{array}$ \\
\hline GRUPO A & 40 & 191 & 18 & 5 & 1 & 9 & 6 & 93 \\
& 11.02 & 52.62 & 4.96 & 1.38 & 0.28 & 2.48 & 1.65 & 25.62 \\
\hline GRUPO B & 11 & 278 & 3 & 2 & 0 & 26 & 5 & 66 \\
& 2.81 & 71.10 & 0.77 & 0.51 & 0.00 & 6.65 & 1.28 & 16.88 \\
\hline
\end{tabular}




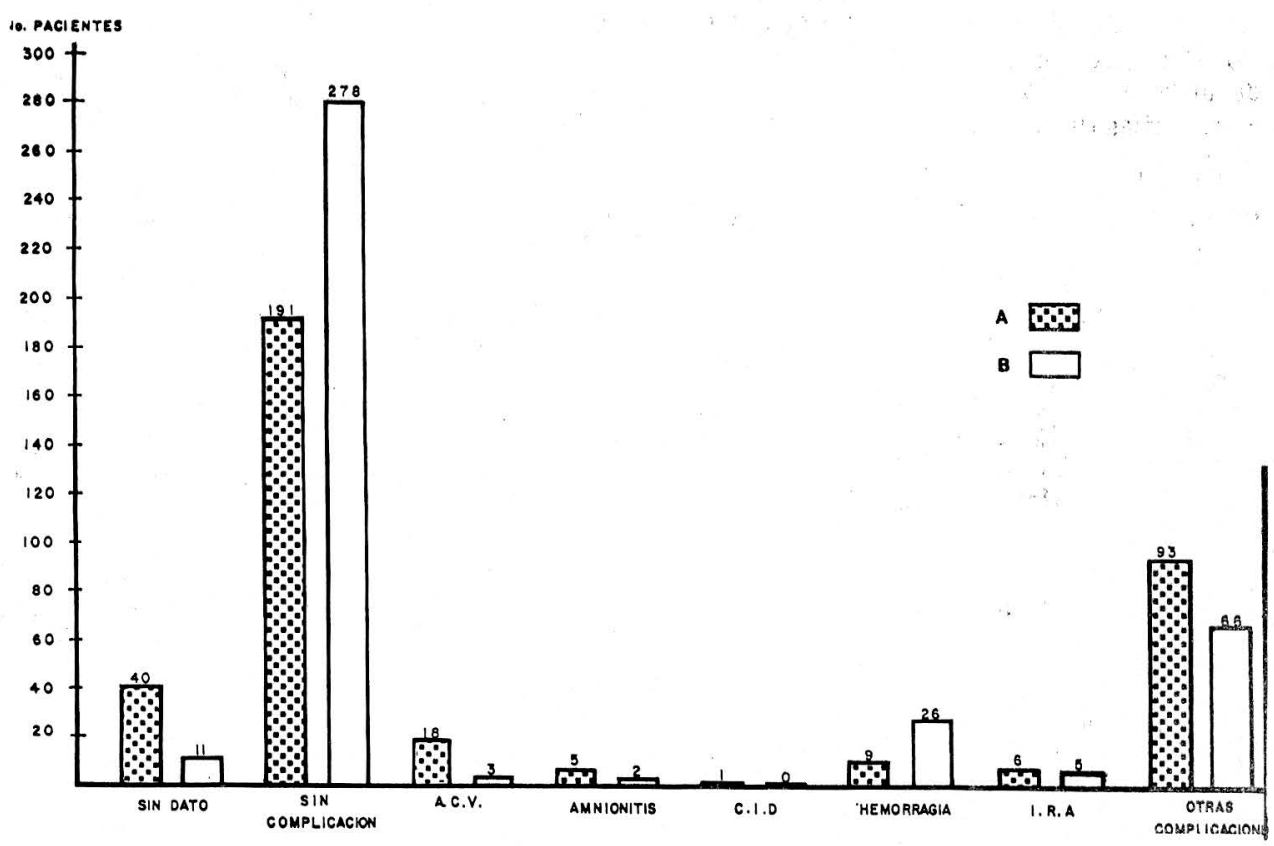

MORTALIDAD PERINATAL

En el cuadro No. 4 se detalla la mortalidad perinatal según ésta haya ocurrido antes o después del ingreso al hospital. Para efectos del análisis de la eficacia de los tratamientos se tendrán en cuenta solamente los casos que ingresaron al

Cuadro No. 4

TRATAMIENTO VS. COMPLICACIONES DEL RECIEN NACIDO

\begin{tabular}{|c|c|c|c|c|c|c|c|}
\hline & № Casos & $\begin{array}{l}\text { MORTALIDAD EX } \\
\text { TRAHOSPITALARIA }\end{array}$ & $\begin{array}{l}\text { MUERTE } \\
\text { ANTEPARTO }\end{array}$ & $\begin{array}{l}\text { MUERTE } \\
\text { INTRAPARTO }\end{array}$ & $\begin{array}{l}\text { MUERTE } \\
\text { NEONATAL }\end{array}$ & $\begin{array}{l}\text { TOTAL INTRA } \\
\text { HOSPITALARIA }\end{array}$ & $\begin{array}{l}\text { MORTALIDA } \\
\text { TOTAL }\end{array}$ \\
\hline \multirow[t]{2}{*}{ GRUPO A } & 363 & 12 & 37 & 22 & 24 & 83 & 95 \\
\hline & & $3.31 \%$ & $10.19 \%$ & $6: 06 \%$ & $6.61 \%$ & $22.83 \%$ & $26.37 \%$ \\
\hline \multirow[t]{4}{*}{ GRUPO B } & 391 & 21 & 16 & 3 & i6 & 35 & 56 \\
\hline & & $5.37 \%$ & $4.09 \%$ & $0.77 \%$ & $4.09 \%$ & $8.95 \%$ & $14.32 \%$ \\
\hline & $x^{2}$ & 1.91 & 10.22 & 16.45 & 4.12 & 26.48 & 27.60 \\
\hline & $\mathrm{p}$ & N.S. & 0.01 & 0.001 & 0.042 & 0.001 & 0.001 \\
\hline
\end{tabular}


Hospital con feto vivo, se discriminará si el fallecimiento ocurre durante el período del anteparto, en el intraparto o durante el período neonatal (siete primeros días de vida).

En el grupo $A$ fallecen 95 fetos $y$ recién nacidos $(26.17 \%)$, en el grupo $B$ fallecen 56 fetos $y$ recién nacidos $(14.32 \%) \quad\left(X^{2}=27.60, p=0.001\right)$, la diferencia es altamente significativa.

Analizadas las cifras de los fetos fallecidos antes del ingreso al hospital se encontraron 12 casos en el grupo $A$ $(3.31 \%)$ y 21 casos en el grupo B $(5.37 \%)$, la diferencia no es significativa $\left(X^{2}=1.91\right)$.

\section{MORTALIDAD PERINATAL INTRAHOSPITALARIA}

Muerte fetal anteparto: en el grupo $A$ mueren 37 fetos $(10.19 \%)$ y en el grupo
B, 16 fetos $(4.09 \%)$, el $X^{2}$ es de 10.22 , $\mathrm{p}=0.01$. La diferencia es significativa.

Muerte fetal intraparto: en el grupo $A$ fallecen 22 fetos $(6.06 \%)$ y en el grupo B, 3 fetos, $(0.77 \%), x^{2}=16.45, p=$ 0.001 . La diferencia estadística es altamente significativa.

Muerte neonatal: En el grupo $A$ fallecen 24 recién nacidos $(6.61 \%)$ y en el grupo B mueren 16 casos $(4.09 \%)$ $X^{2}=4.12, p=0.042$. La diferencia es significativa.

La mortalidad total intrahospitalaria en el grupo $A$ es de $83 \operatorname{casos}(22,83 \%)$ $y$ en el $B$ es de 35 casos $(8,95 \%)$, $X^{2}=26.48, p=0.001$, se aprecia una notoria y significativa disminución de la mortalidad en el grupo B, especialmente debido a la reducción de la mortalidad en el período ante e intraparto.

MORTALIDAD FETAL Y NEONATAL TOTAL SEGUN EL PESO

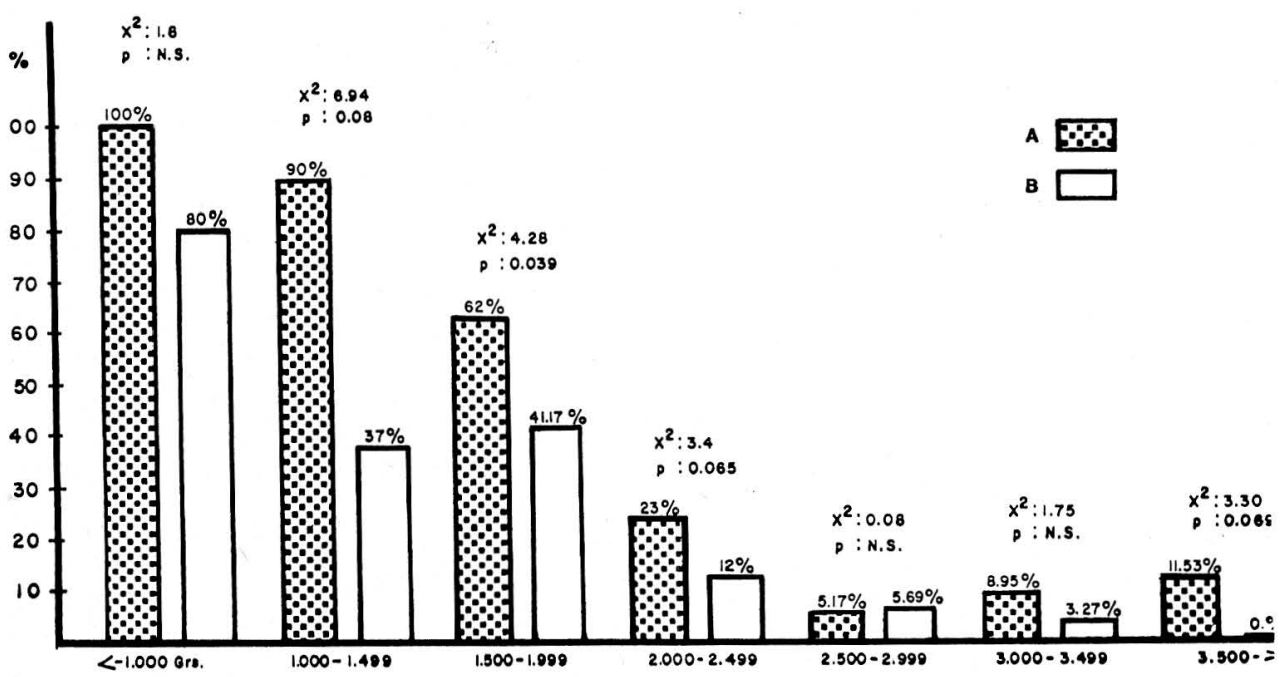




\section{MORTALIDAD PERINATAL SEGUN PESO DEL RECIEN NACIDO}

En el cuadro No. 5 se relaciona la mortalidad total del producto según el peso.

R.N. menor de $1.000 \mathrm{gr}$. la mortalidad en el grupo $A$ es del $100 \%$ y en el grupo $B$ es del $80 \%\left(X^{2}=1.8, p=\right.$ N.S. $)$.

R.N. de 1.000 a 1.499 grs. la mortalidad en el grupo $A$ es del $90 \%$ y en el $B$ del $37 \%,\left(X^{2}=6.94, p=\right.$ N.S. $)$.

R.N. de 1.500 a 1.999 grs. La mortalidad del grupo $A$ fue del $62 \%$ y en el grupo $B$ del $41.97 \% \quad\left(X^{2}=4.28\right.$ $\mathrm{p}=0.039$ ).

Hay disminución significativa de la mortalidad en este grupo.
R.N. de 2.000 a 2.499 grs, la mortalidad en el grupo A fue del $23 \%$ y en el grupo $B$ del $12 \%\left(X^{2}=3.4, p=0.06\right)$.

A pesar que la reducción en porcentaje es alta, las cifras no son significativas.

R.N. de 2.500 a 2.999 grs, Ia mortalidad en el grupo $A$ es de 5.17 y en el grupo $B$ del $5.69\left(X^{2}=0.08\right.$, p.N.S.).

R.N. de 3.000 a 3.499 grs. la mortalidad en el grupo $A$ es del $8.95 \%$ y en el grupo $B 3.27 \%\left(X^{2}=1.75, p=N . S.\right)$.

A pesar que la reducción en porcentaje es notoria, no hay significancia estadística.

R.N. mayor de 3.500 grs. la mortalidad es del $11.53 \%$ en el grupo $\mathrm{A}$ y del $0 \%$ en el $\mathrm{B}\left(\mathrm{X}^{2}=3.30, \mathrm{p}=0.069\right)$.

Cuadro No. 5

MORTALIDAD FETAL Y NEONATAL TOTAL

GRUPO A

GRUPO B

\begin{tabular}{|ccccccccc|}
\hline \multicolumn{1}{c}{ MORTALIDAD } & MORTALIDAD & & \\
\hline Peso & NN Casos & NN Casos & $\%$ & No Casos & No Casos & $\%$ & X2 & P \\
\hline 1.000 grs & 8 & 8 & 100 & 10 & 8 & 80 & 1.8 & N.S. \\
$1.000-1.499$ & 10 & 9 & 90 & 16 & 6 & 37 & 6.94 & 0.08 \\
$1.500-1.999$ & 45 & 28 & 62 & 51 & 21 & 41.17 & 4.28 & 0.039 \\
$2.000-2.499$ & 77 & 18 & 23 & 95 & 12 & 12.63 & 3.4 & 0.065 \\
$2.500-2.999$ & 116 & 6 & 5.17 & 123 & 7 & 5.69 & 0.08 & N.S. \\
$3.000-3.499$ & 67 & 6 & 8.95 & 61 & 2 & 3.27 & 1.75 & N.S. \\
3.500 & 26 & 3 & 11.53 & 27 & 0 & 0.0 & 3.30 & 0.059 \\
\hline
\end{tabular}

No hay diferencia significativa.

Para evaluar la eficacia de las conductas y tratamientos en relación con la mortalidad fetal relacionado con el peso se analizaron los datos de los casos en los cuales el feto ingresa vivo al servicio, los resultados son los siguientes. Cuadro No. 6.
La mortalidad intrahospitalaria está correlacionada significativamente con el peso del R.N. $(r=0.945, p=0.001)$.

R.N. menor de 1.000 grs. mortalidad en el grupo $A$ es del $100 \%$, en el grupo $B$ es del $75 \%\left(X^{2}=1.75, p=N . S.\right)$.

R.N. de 1.000 a 1.499 grs. mortalidad en el grupo $A$ es del $90 \%$ y en el grupo 


\section{MORTALIDAD PERINATAL GLOBAL}

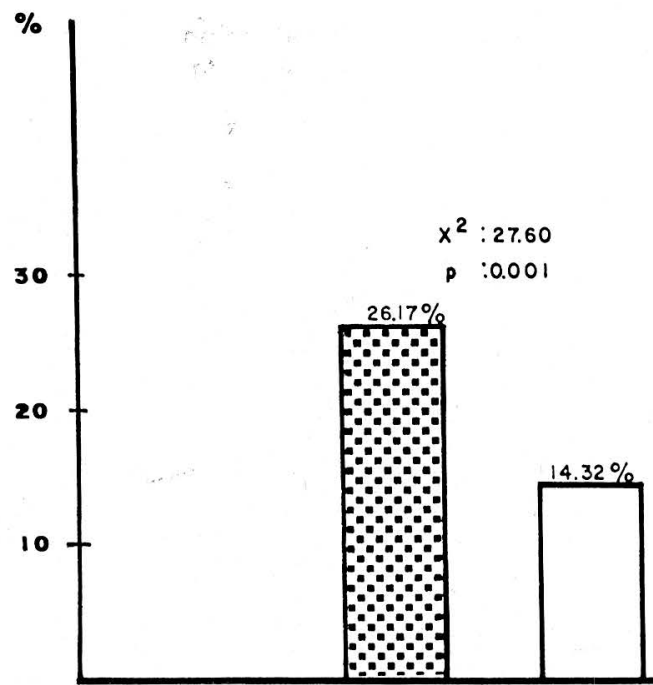

A $B$

B

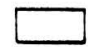

B es del $23 \%\left(X^{2}=10.14, p=0.001\right)$. La reducción es significativa.

R.N. de 1.500 a 1.999 grs. la mortalidad en el grupo $A$ es del $59.5 \%$ y en el grupo $\mathrm{B}$ del $30 \% \quad\left(X^{2}=7.37, \mathrm{p}=\right.$ 0.007). La reducción es significante.
R.N. de 2.000 a 2.499 grs. la mortalidad en el grupo $A$ es del $23 \%$ y en el grupo $\mathrm{B}$ del $7.7 \% \quad\left(\mathrm{X}^{2}=6.08, \mathrm{p}=\right.$ 0.01 ), la reducción es significante.

R.N. de 2.500 a 2.999 grs. la mortalidad en el grupo $A$ es del $5.17 \%$ y en el

Cuadro No. 6

MORTALIDAD FETAL Y NEONATAL INTRAHOSPITALARIA

\begin{tabular}{|cccccccccc|}
\hline \multicolumn{1}{c|}{ MORTALIDAD } \\
\hline Peso & No Casos & No Casos & $\%$ & NN Casos & No Casos & $\%$ & X2 & p \\
\hline 1.000 grs. & 6 & 6 & 100 & 8 & 6 & 75 & 1.75 & 0.08 \\
$1.000-1.499$ & 10 & 9 & 90 & 13 & 3 & 23 & 10.14 & 0.001 \\
$1.500-1.999$ & 42 & 25 & 59.5 & 43 & 13 & 30.0 & 7.37 & 0.007 \\
$2.000-2.499$ & 77 & 18 & 23 & 90 & 7 & 7.7 & 6.08 & 0.014 \\
$2.500-2.999$ & 116 & 6 & 5.17 & 120 & 4 & 3.33 & 0.4 & N.S. \\
$3.000-3.499$ & 64 & 3 & 4.68 & 61 & 2 & 3.27 & 0.16 & N.S. \\
3.500 & 24 & 1 & 4.16 & 27 & 0 & 0.0 & 0.14 & N.S. \\
\hline
\end{tabular}


MORTALIDAD PERINATAL INTRAHOSPITALARIA

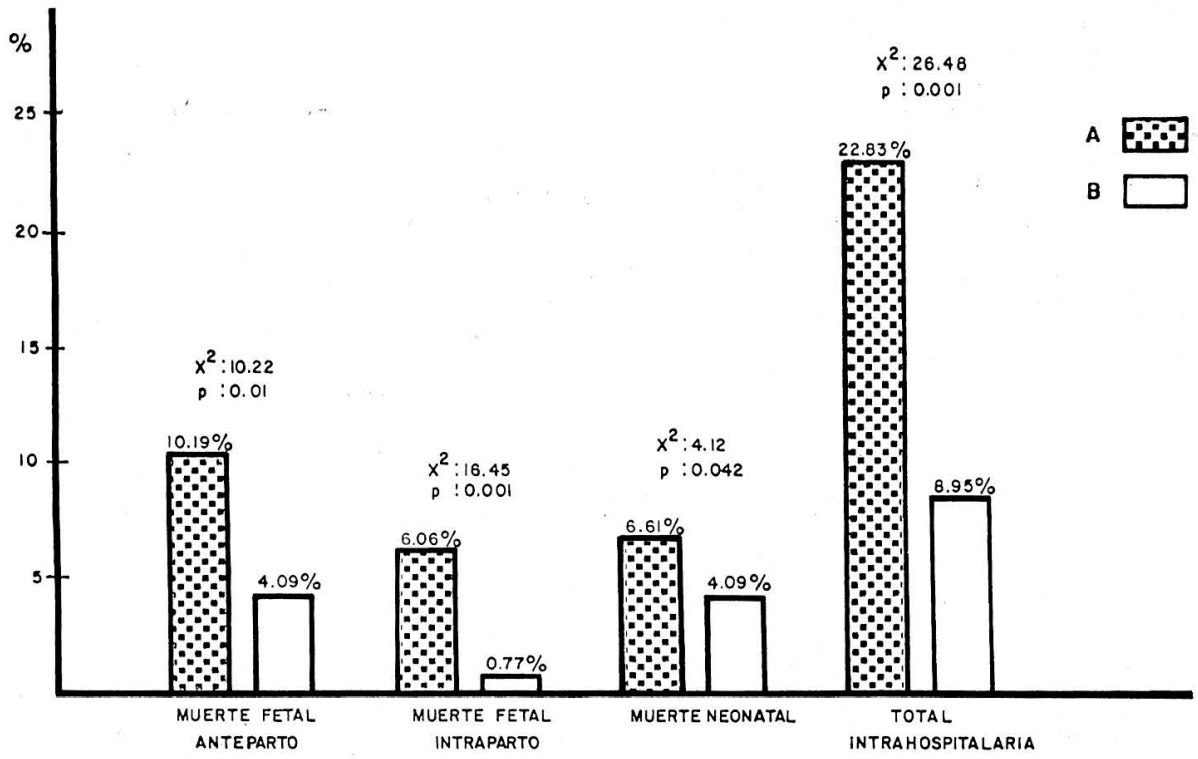

MORTALIDAD FETAL Y NEONATAL INTRAHOSPITALARIA

SEGUN EL PESO

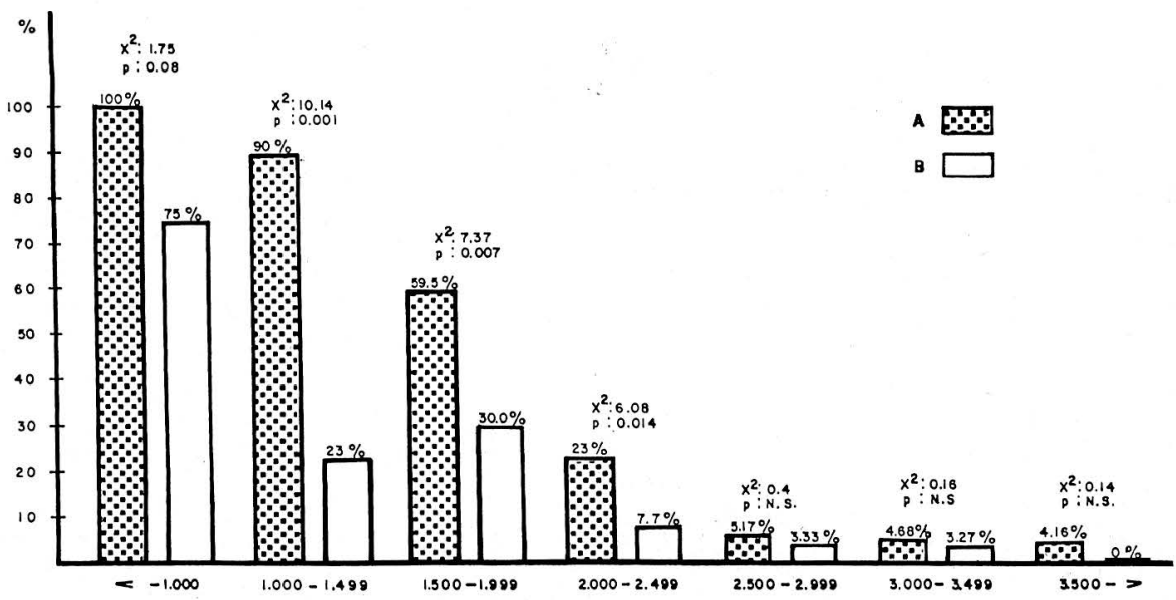


grupo $B$ del $3.33 \%(X 2=0.4, p=$ N.S.).

R.N. de 3.000 a 3.499 grs. la mortalidad en al grupo $A$ fue del $4.68 \%$ y en el grupo $\mathrm{B}$ del $3.27 \%\left(X^{2}=0.16, \mathrm{p}=\right.$ N.S.

R.N. mayor de 3.500 grs. la mortalidad en el grupo A fue del $4.16 \%$ y en el grupo $B 0 \%\left(X^{2}=0.14, p=N . S.\right)$.

En las cifras analizadas se aprecia que se ha logrado reducción significativa de la mortalidad en las pacientes del grupo $B$ en los niños prematuros. No hay reducción significativa entre los niños mayores de $2.500 \mathrm{grs}$.

Los mejores resultados obtenidos en el grupo B no pueden atribuirse al uso de una determinada droga sino al conjunto de normas en el manejo de las pacientes y una más acertada decisión respecto a la fecha en la cual se desembaraza a la paciente y la vía por la cual se hace este procedimiento.

Aún existen algunas fallas en el manejo del recién nacido, una mayor reduc- ción podría lograrse si en esta sección se iniciaran pautas de cuidado intensivo más rigurosas.

\section{ANALISIS DE MORTALIDAD SEGUN EL TIPO DE PARTO}

En el cuadro No. 7 se registra la mortalidad general relacionada al tipo de parto, análisis que se hace para discriminar si la diferencia de conductas pudo haber influenciado en los resultados finales.

\section{PARTO POR VIA VAGINAL}

Parto espontáneo, se presentó en 209 casos del grupo $A$ entre los cuales hubo 50 casos de mortalidad perinatal (23. $9 \%$. En el grupo B 116 partos fueron espontáneos entre los cuales fallecieron 8 casos $(6.89 \%$ ). La diferencia del Chi cuadrado es de 14.75 y el $p=0.01$.

El parto fue inducido $y$ atendido por vía vaginal en 28 casos del grupo $A$ entre los cuales fallecieron 9 niños, $(32.14 \%)$. En el grupo B, 89 casos fueron inducidos y solamente 9 niños, fa-

\section{Cuadro No. 7}

MORTALIDAD FETAL Y NEONATAL INTRAHOSPITALARIA SEGUN EL TIPO DE PARTO

\begin{tabular}{|c|c|c|c|c|c|c|c|c|}
\hline \multicolumn{4}{|c|}{ GRUPO A } & & \multicolumn{4}{|c|}{ GRUPO B } \\
\hline & & $\begin{array}{c}\text { MORTALID } \\
\text { R.N. }\end{array}$ & & & $\begin{array}{c}\text { MORTALI } \\
\text { R.N. }\end{array}$ & DAD & & \\
\hline Partos & № Casos & № Casos & $\%$ & № Casos & № Casos & $\%$ & $\times 2$ & $p$ \\
\hline Espontáneo & 209 & 50 & 23.9 & 116 & 8 & 6.89 & 14.75 & 0.001 \\
\hline Induc.+ Vag. & 28 & 9 & 32.14 & 89 & 9 & 10.0 & 7.94 & 0.005 \\
\hline Instr. Vag. & 28 & 1 & 3.57 & 44 & 5 & 11.3 & 1.36 & N.S. \\
\hline TOTAL & 265 & 60 & 22.64 & 249 & 22 & 8.33 & 18.24 & 0.001 \\
\hline Induc. + Ces. & 9 & 0 & 0.0 & 13 & 1 & 7.69 & 0.7 & N.S. \\
\hline Cesárea & 82 & 20 & 24.39 & 127 & 12 & 9.44 & 8.57 & 0.003 \\
\hline$T O T A L$ & 91 & 20 & 21.97 & 140 & 13 & 9.28 & 7.25 & 0.007 \\
\hline
\end{tabular}


llecieron $(10 \%)$. La diferencia es significativa: Chi2 $=7.94, p=0.005$.

El parto por vía vaginal fue instrumentado en 28 casos del grupo $A$ y hubo un caso de mortalidad $(3.57 \%)$. En el grupo $\mathrm{B}$ se instrumentaron 44 partos $\mathrm{y}$ hubo mortalidad en $5(11.3 \%)$. No se discriminó si la maniobra fue hecha en casos con mortalidad antenatal o no. No hay diferencia estadística en estos datos. Chi2 $=1.36, p=$ N.S

En total hubo 265 partos vaginales en el grupo $A$ en el cual fallecieron 60 niños $(22.64 \%)$ y en el grupo $B$ hubo 249 casos y fallecieron en total solo 22 niños $(8.33 \%)$. La diferencia de mortalidad favorece significativamente a los casos del grupo B. Chi2 $=18.24, p=$ 0.001 .

\section{CESAREA}

En el grupo A, 9 casos fueron inducidos $y$ finalmente fueron intervenidos por cesárea, no hubo niños muertos en este grupo de pacientes. En el grupo B, 13 casos fueron inducidos $y$ posteriormente intervenidos con cesárea; de ellos uno presentó muerte del recién nacido ( 7 . $69 \%$ ). No hay diferencia significativa: Chi2 $=0.7, p=$ N.S.

En el grupo $A$ se practicaron 82 cesáreas y la mortalidad perinatal se presentó en $20(24.39 \%)$, en el grupo B se practicaron 127 cesáreas y la mortalidad perinatal se observó en 12 casos $(9.44 \%)$. La diferencia es altamente significativa, Chi2 $=8.57, \mathrm{p}=0.003$.

El total de pacientes intervenidas en el grupo A fue de 91 casos, en los cuales la

\section{COMPLICACIONES PERINATALES VS. TIPO DE PARTO EN EL TRATAMIENTO ANTERIOR}

(A)

\begin{tabular}{|c|c|c|c|c|c|c|c|c|c|}
\hline & - In Dato & Eapontanto & Imoucloor vaoinay & ceonatea & clesar. nostmonse & imatrumentado & InOUCioo retoan. & $0 . T \cap 0$ & TErAL \\
\hline $\begin{array}{l}\text { FRECUENCIA } \\
\% \times \text { COLUMNA }\end{array}$ & $2500^{1}$ & $\begin{array}{r}9 \\
4.31\end{array}$ & $\begin{array}{r}0 \\
0.00\end{array}$ & 4.68 & $\begin{array}{r}0 \\
0.00\end{array}$ & $\begin{array}{r}0 \\
0.00\end{array}$ & $\begin{array}{r}0 \\
0.00\end{array}$ & $\begin{array}{r}0 \\
0.00^{\prime}\end{array}$ & 14 \\
\hline SIN COMPLICACION & 25.00 & $\begin{array}{r}143 \\
69.38\end{array}$ & $\begin{array}{r}16 \\
57.14\end{array}$ & $\begin{array}{r}55 \\
67.07\end{array}$ & $\begin{array}{r}0 \\
0.00\end{array}$ & $\begin{array}{r}26 \\
22.86\end{array}$ & $100.00^{9}$ & 100.00 & 254 \\
\hline $\begin{array}{c}\text { MUERTE } \\
\text { EXTRAHOSPITALARIA }\end{array}$ & $\begin{array}{r}0 \\
.0 .00\end{array}$ & 2.39 & $10.71^{3}$ & $\begin{array}{r}3 \\
3.68\end{array}$ & 0.00 & 3.57 & $\begin{array}{r}0 \\
0.00\end{array}$ & 0.00 & 12 \\
\hline $\begin{array}{l}\text { MUERTE } \\
\text { INTRA HOSPITALARIA } \\
\text { ANTEPARTO }\end{array}$ & $\begin{array}{r}2 \\
50.00\end{array}$ & $\begin{array}{r}20 \\
0.57\end{array}$ & $\begin{array}{r}8 \\
28.57\end{array}$ & $\begin{array}{r}7 \\
8.54\end{array}$ & $\begin{array}{r}0 \\
0.00\end{array}$ & $\begin{array}{r}0 \\
0.00\end{array}$ & $\begin{array}{r}0 \\
0.00\end{array}$ & $\begin{array}{r}0 \\
0.00\end{array}$ & 37 \\
\hline $\begin{array}{c}\text { MUERTE } \\
\text { INTRAHOSPITALARIA } \\
\text { INTRAPARTO }\end{array}$ & $\begin{array}{r}0 \\
0.00\end{array}$ & $\begin{array}{r}17 \\
8.13\end{array}$ & $\begin{array}{r}0 \\
0.00\end{array}$ & 4.88 & $\begin{array}{r}0 \\
0.00\end{array}$ & $\begin{array}{r}1 \\
3.57\end{array}$ & $\begin{array}{r}0 \\
0.00\end{array}$ & $\begin{array}{r}0 \\
0.00\end{array}$ & 22 \\
\hline $\begin{array}{l}\text { MUERTE } \\
\text { NEONATAL }\end{array}$ & $\begin{array}{r}0 \\
0.00\end{array}$ & $\begin{array}{r}13 \\
6.22\end{array}$ & $\begin{array}{r}1 \\
3.57\end{array}$ & 10.98 & $100.00^{1}$ & $\begin{array}{r}0 \\
0.00\end{array}$ & 0.00 & $\begin{array}{r}0 \\
0.00\end{array}$ & 24 \\
\hline TOTAL & $\begin{array}{r}4 \\
1.10^{4} \\
\end{array}$ & $\begin{array}{r}209 \\
57.58 \\
\end{array}$ & $\begin{array}{r}28 \\
7.71 \\
\end{array}$ & $\begin{array}{r}82 \\
22.5 .9 \\
\end{array}$ & $\begin{array}{r}1 \\
0.28 \\
\end{array}$ & $\begin{array}{r}28 \\
7.71 \\
\end{array}$ & $\begin{array}{r}9 \\
2.48 \\
\end{array}$ & $\begin{array}{r}2 \\
0.53 \\
\end{array}$ & $\begin{array}{r}363 \\
10000 \\
\end{array}$ \\
\hline
\end{tabular}


COMPLICACIONES PERINATALES VS. TIPO DE PARTO

EN EL TRATAMIENTO ACTUAL

(B)

\begin{tabular}{|c|c|c|c|c|c|c|c|c|c|}
\hline$\Gamma$ & DIN DATO & ESPONTANEO & INOUCIDO+ VAOINAL & CESAREA & CEsan. NOST.MOET & IN \&TAUNENTADO & woweioo reteun. & OT: & reral \\
\hline $\begin{array}{l}\text { FRECUENCIA } \\
\text { \% X COLUMNA }\end{array}$ & 100.00 & $\begin{array}{r}3 \\
2.59\end{array}$ & $\begin{array}{r}0 \\
0.00\end{array}$ & $\begin{array}{r}0 \\
0.00\end{array}$ & 0 & $\begin{array}{r}0 \\
0.00\end{array}$ & $\begin{array}{r}0 \\
0.00\end{array}$ & $100.00^{1}$ & 5 \\
\hline SIH COMPLICACION & $\begin{array}{r}0 \\
0.00\end{array}$ & $\begin{array}{r}103 \\
88.79\end{array}$ & $\begin{array}{r}66 \\
74.16\end{array}$ & $\begin{array}{r}112 \\
88.10\end{array}$ & $\begin{array}{l}0 \\
.\end{array}$ & $\begin{array}{r}38 \\
636\end{array}$ & $\begin{array}{r}11 \\
84.62\end{array}$ & $\begin{array}{r}0 \\
0.00\end{array}$ & 330 \\
\hline $\begin{array}{c}\text { MUERTE } \\
\text { EXTRAHOSPITALARIA }\end{array}$ & $\begin{array}{r}0 \\
0.00\end{array}$ & $\begin{array}{r}2 \\
1.72\end{array}$ & $\begin{array}{r}14 \\
15.73\end{array}$ & 2.36 & $\begin{array}{l}0 \\
.\end{array}$ & $\begin{array}{r}1 \\
2.27\end{array}$ & $\begin{array}{r}1 \\
7.60\end{array}$ & $\begin{array}{r}0 \\
0.00\end{array}$ & 21 \\
\hline $\begin{array}{c}\text { MUERTE } \\
\text { INTRAHOSPITALARIA } \\
\text { ANTEPARTO }\end{array}$ & $\begin{array}{r}0 \\
0.00\end{array}$ & 4.31 & $\begin{array}{r}8 \\
8.99\end{array}$ & $\begin{array}{r}1 \\
0.79\end{array}$ & $\begin{array}{l}0 \\
.\end{array}$ & $\begin{array}{r}2 \\
4.55\end{array}$ & $\begin{array}{r}0 \\
000\end{array}$ & $\begin{array}{r}0 \\
0.00\end{array}$ & 16 \\
\hline $\begin{array}{c}\text { MUERTE } \\
\text { INTKA HOSPITALARIA } \\
\text { INTR APARTO }\end{array}$ & $\begin{array}{r}0 \\
0.00\end{array}$ & $\begin{array}{r}2 \\
1.72\end{array}$ & $\begin{array}{r}0 \\
0.00\end{array}$ & $\begin{array}{r}0 \\
0.00\end{array}$ & $\begin{array}{l}0 \\
.\end{array}$ & $\begin{array}{r}1 \\
227\end{array}$ & $\begin{array}{r}0 \\
0.00\end{array}$ & $\begin{array}{r}0 \\
0.00\end{array}$ & 3 \\
\hline $\begin{array}{l}\text { MUERTE } \\
\text { NEONATAL }\end{array}$ & $\begin{array}{c}0 \\
0.00\end{array}$ & $\begin{array}{r}1 \\
0.86\end{array}$ & $\begin{array}{r}1 \\
1.12\end{array}$ & $\begin{array}{r}11 \\
8.66\end{array}$ & $\begin{array}{l}0 \\
.\end{array}$ & $\begin{array}{r}2 \\
4.55\end{array}$ & $\begin{array}{r}1 \\
7.69\end{array}$ & $\begin{array}{r}0 \\
0.00\end{array}$ & 16 \\
\hline TOTAL & $\begin{array}{r}1 \\
0.26\end{array}$ & $\begin{array}{r}116 \\
29.67\end{array}$ & $\begin{array}{r}89 \\
22.76\end{array}$ & $\begin{array}{r}127 \\
32.48\end{array}$ & . & $\begin{array}{r}44 \\
11.25\end{array}$ & $\begin{array}{r}18 \\
3.32\end{array}$ & $\begin{array}{r}1 \\
0.26\end{array}$ & $\begin{array}{r}391 \\
100.00\end{array}$ \\
\hline
\end{tabular}

MORTALIDAD FETAL Y NEONATAL INTRAHOSPITALARIA SEGUN EL TIPO DE PARTO

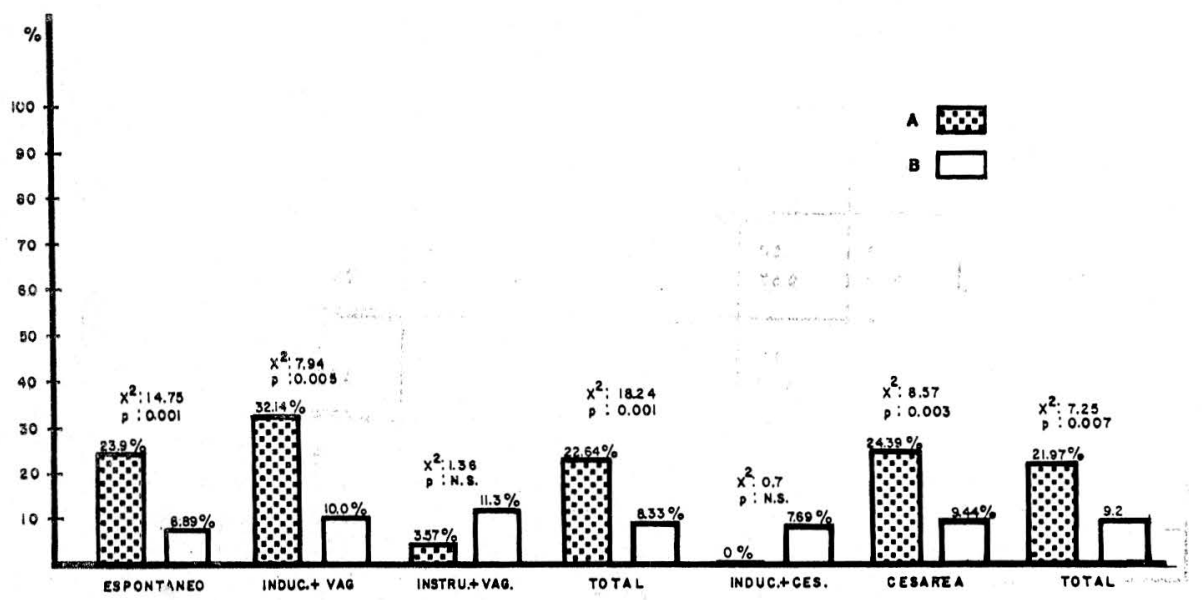


mortalidad perinatal se presentó en 20 casos $(21.9 \%)$. En el grupo B se intervinieron 140 pacientes en las cuales 13 niños fallecen $(9.28 \%)$. La diferencia en la mortalidad es significativa: Chi2 = 7.25, $p=0.007$.

\section{MORTALIDAD FETAL $Y$ NEONATAL RELACIONADA CON EL DİAGNOSTICO}

\section{ENFERMEDAD HIPERTENSIVA INDUCIDA POR EL EMBARAZO}

En 116 casos de preeclampsia del grupo A hay mortalidad en 11 casos $(9.49 \%)$ y en 122 casos del grupo B" la mortalidad se presenta en 4 casos $(3.27 \%)$. Las cifras favorecen al grupo B. $\mathrm{Chi}=23,87, \mathrm{p}=0.049$. (Ver cuadro No. 8).

En 78 casos con inminencia de eclampsia, en el grupo A fallecen 16 niños $(20.51 \%)$, en 54 casos del grupo
B fallecen 5 niños $(9.25 \%)$. Las cifras estadísticas favorecen al grupo B. Chi2 $=4.30, \mathrm{p}=0.038$.

En 62 casos de eclampsia del grupo $A$ fallecieron 20 niños $(32.25 \%)$ y en 76 casos de eclampsia del grupo B fallecen 10 niños $(13.15 \%)$. Las cifras tienen una diferencia estadística muy alta: Chi2 $=7.32 \mathrm{p}=0.007$.

En total 256 casos de enfermedad hipertensiva inducida por el embarazo del grupo A fallecen 47 niños $(18.35 \%)$ y en 252 casos de enfermedad hipertensiva inducida por el embarazo del grupo $B$ fallecen 19 niños $(7.53 \%)$. El estudio de significancia favorece al grupo B: Chi2 $=13.15, p=0.001$.

\section{ENFERMEDAD VASCULAR HIPERTENSIVA CRONICA}

En 26 casos de EVHC con HIE sobreagregada fallecen 5 niños $(19.23 \%)$ en el grupo A. En 60 casos del grupo B con

Cuadro No. 8

MORTALIDAD FETAL Y NEONATAL INTRAHOSPITALARIA SEGUN EL DIAGNOSTICO

\begin{tabular}{|c|c|c|c|c|c|c|c|c|c|c|c|c|}
\hline \multirow[b]{2}{*}{ Diagnóstico } & & \multicolumn{3}{|c|}{ MORTALIDAD R.N. } & \multicolumn{7}{|c|}{ MORTALIDAD R.N. } \\
\hline & $\mathrm{N}=$ & - Casos & № & Casos & $\%$ & № & Casos & № & Casos & $\%$ & $\times 2$ & $p$ \\
\hline Preeclampsia (HIE) & & 116 & & 11 & 9.48 & & 122 & & 4 & 3.27 & 3.87 & 0.049 \\
\hline Inm.Eclampsia (IE) & & 78 & & 16 & 20.51 & & 54 & & 5 & 9.25 & 4.30 & 0.038 \\
\hline Eclampsia & & 62 & & 20 & 32.25 & & 76 & & 10 & 13.15 & 7.32 & 0.007 \\
\hline TOTAL & & 256 & & 47 & 18.35 & & 252 & & 19 & 7.53 & 13.15 & 0.001 \\
\hline EVHC + P.E.* $\left(H I E^{\star}\right)$ & . & 26 & & 5 & 19.23 & & 60 & & 6 & 10.0 & 1.38 & N.S. \\
\hline$E V H C+I . E . \star$ & & 47 & & 18 & 38.29 & & 44 & & 3 & 6.81 & 12.68 & 0.001 \\
\hline$E V H C+E^{\star}$ & & 14 & & 3 & 21.42 & & $2 C$ & & 6 & 30.0 & 0.31 & N.S. \\
\hline EVHC & & 17 & & 9 & 52.94 & & 11 & & 0 & 0.0 & N.S. & N.S. \\
\hline TOTAL & 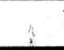 & 104 & & 35 & $33: 65$ & & 135 & & 15 & 11.11 & 18.04 & 0.001 \\
\hline $\begin{array}{l}\text { H.I.E. Hipertensión } \\
\text { Embarazo }\end{array}$ & ucid & & & Preecl & ampsia & I.E. Inmin & nencia & de $E c$ & i & E. & Eclamps & \\
\hline
\end{tabular}


diagnóstico similar fallecen 6 niños $(10 \%)$. La diferencia no es significativa, Chi2 $=1.38, p=$ N.S.

En 47 casos del grupo A con EVHC con inminencia de eclampsia sobreagregada fallecen 18 niños $(38.29 \%)$ $y$ en 44 casos del grupo $B$ con el mismo diagnóstico fallecen 3 niños $(6.81 \%)$ lo que significa una diferencia altamente notoria, $\mathrm{Chi} 2=12.68 \mathrm{p}=0.001$.

En 14 casos de EVHC con eclampsia sobreagregada en el grupo $A$ fallecen 3 niños $(21.42 \%)$ y en 20 casos del grupo $B$ con el mismo diagnóstico fallecen 6 niños $(30 \%)$. La diferencia no es significativa, Chi2 $=31, p=$ N.S.

En 17 casos de EVHC solo fallecen 9 niños del grupo A $(52.94 \%)$ y en el grupo $B$ entre 20 casos fallecen 6 niïos. La cifra no da diferencia significativa.

En total entre 104 casos del grupo $A$ con EVHC sola o con HIE sobreagregada se encuentran 35 niños fallecidos ( 33 . $65 \%$ ) y entre 135 pacientes con igual diagnóstico en el grupo B fallecen 15 niños $(11.11 \%)$ lo que representa una gran diferencia estadística a favor del grupo B. Chi2 $=18.04, p=0.001$.

\section{COMPLICACIONES PERINATALES VS. DIAGNOSTICO EGRESO}

EN EL TRATAMIENTO ANTERIOR

(A)

\begin{tabular}{|c|c|c|c|c|c|c|c|c|c|c|}
\hline & SIN DATO & P.E & I. E & E. & EVHC + P.E & EVHC + I.E. & EVHG + E & E.Y.H. C. & OTRO & TOTAL \\
\hline $\begin{array}{l}\text { FRECUENCIA } \\
\% \\
\% \times \text { FILA } \\
\% \times \text { COLUMNA } \\
\end{array}$ & $\begin{array}{l}0 \\
0.00 \\
0.00 \\
0.00\end{array}$ & $\begin{array}{r}5 \\
1.38 \\
35.71 \\
4.31 \\
\end{array}$ & $\begin{array}{r}2 \\
0.55 \\
14.29 \\
2.56 \\
\end{array}$ & $\begin{array}{r}4 \\
1.10 \\
28.57 \\
6.45 \\
\end{array}$ & $\begin{array}{r}0 \\
0.00 \\
0.00 \\
0.00\end{array}$ & $\begin{array}{ll} & 1 \\
0.28 \\
7.14 \\
2.13 \\
\end{array}$ & $\begin{array}{r}2 \\
0.55 \\
14.29 \\
1429 \\
\end{array}$ & $\begin{array}{r}0 \\
0.00 \\
0.00 \\
0.00 \\
\end{array}$ & $\begin{array}{r}0 \\
0.00 \\
0.00 \\
0.00\end{array}$ & $\begin{array}{r}14 \\
3.86\end{array}$ \\
\hline SIN COMPLICACION & $\begin{array}{r}1 \\
0.28 \\
0.39 \\
100.00 \\
\end{array}$ & $\begin{array}{r}97 \\
26.72 \\
38.19 \\
83.62 \\
\end{array}$ & $\begin{array}{r}58 \\
15.98 \\
22.83 \\
74.36 \\
\end{array}$ & $\begin{array}{r}35 \\
9.64 \\
13.78 \\
56.45 \\
\end{array}$ & $\begin{array}{r}20 \\
5.51 \\
7.87 \\
76.92 \\
\end{array}$ & $\begin{array}{r}26 \\
7.16 \\
10.24 \\
55.32 \\
\end{array}$ & $\begin{array}{r}8 \\
2.20 \\
3.15 \\
57.14 \\
\end{array}$ & $\begin{array}{r}8 \\
2.20 \\
3.15 \\
47.06 \\
\end{array}$ & $\begin{array}{r}1 \\
0.28 \\
0.39 \\
50.00 \\
\end{array}$ & $\begin{array}{r}254 \\
69.97\end{array}$ \\
\hline $\begin{array}{c}\text { MUERTE } \\
\text { EXTRAHOSPITALARIA }\end{array}$ & $\begin{array}{r}0 \\
0.00 \\
0.00 \\
0.00\end{array}$ & $\begin{array}{r}3 \\
0.83 \\
25.00 \\
2.59 \\
\end{array}$ & $\begin{array}{r}2 \\
0.53 \\
16.67 \\
2.56 \\
\end{array}$ & $\begin{array}{r}3 \\
0.83 \\
25.00 \\
4.84 \\
\end{array}$ & $\begin{array}{r}1 \\
0.28 \\
8.33 \\
3.85 \\
\end{array}$ & $\begin{array}{r}2 \\
0.55 \\
16.67 \\
4.26\end{array}$ & $\begin{array}{r}1 \\
0.28 \\
8.33 \\
7.14 \\
\end{array}$ & $\begin{array}{l}0 \\
0.00 \\
0.00 \\
0.00\end{array}$ & $\begin{array}{r}0 \\
0.00 \\
0.00 \\
0.00\end{array}$ & $\begin{array}{r}12 \\
3.31\end{array}$ \\
\hline $\begin{array}{c}\text { MUERTE } \\
\text { INTRAHOSPITALARIA } \\
\text { ANTEPARTO }\end{array}$ & $\begin{array}{r}0 \\
0.00 \\
0.00 \\
0.00 \\
\end{array}$ & $\begin{array}{r}3 \\
0.83 \\
8.11 \\
2.59 \\
\end{array}$ & $\begin{array}{r}7 \\
1.93 \\
18.92 \\
8.97 \\
\end{array}$ & $\begin{array}{r}9 \\
2.48 \\
24.32 \\
14.52 \\
\end{array}$ & $\begin{array}{r}3 \\
0.63 \\
8.11 \\
11.54 \\
\end{array}$ & $\begin{array}{r}8 \\
2.20 \\
21.62 \\
17.02 \\
\end{array}$ & $\begin{array}{r}2 \\
0.55 \\
5.41 \\
14.29 \\
\end{array}$ & $\begin{array}{r}4 \\
1.10 \\
10.81 \\
23.53 \\
\end{array}$ & $\begin{array}{r}1 \\
0.28 \\
2.70 \\
50.00 \\
\end{array}$ & $\begin{array}{r}37 \\
10.19\end{array}$ \\
\hline $\begin{array}{c}\text { MUERTE } \\
\text { INTRAHOSPITALARIA } \\
\text { INTRAPARTO }\end{array}$ & $\begin{array}{r}0 \\
0.00 \\
0.00 \\
0.00\end{array}$ & $\begin{array}{r}6 \\
1.65 \\
27.27 \\
5.17\end{array}$ & $\begin{array}{r}6 \\
1.65 \\
27.27 \\
7.69\end{array}$ & $\begin{array}{r}4 \\
1.10 \\
18.18 \\
6.45 \\
\end{array}$ & $\begin{array}{r}1 \\
0.28 \\
4.55 \\
3.85 \\
\end{array}$ & $\begin{array}{r}3 \\
0.83 \\
13.64 \\
6.38 \\
\end{array}$ & $\begin{array}{r}1 \\
0.28 \\
4.55 \\
7.14 \\
\end{array}$ & $\begin{array}{r}4 \\
0.28 \\
4.55 \\
5.88 \\
\end{array}$ & $\begin{array}{r}0 \\
0.00 \\
0.00 \\
0.00 \\
\end{array}$ & $\begin{array}{r}22 \\
8.08\end{array}$ \\
\hline $\begin{array}{l}\text { MUERTE } \\
\text { NEONATAL }\end{array}$ & $\begin{array}{r}0 \\
0.00 \\
0.00 \\
0.00 \\
\end{array}$ & $\begin{array}{r}2 \\
0.53 \\
6.33 \\
1.72 \\
\end{array}$ & $\begin{array}{r}3 \\
0.83 \\
12.50 \\
3.85 \\
\end{array}$ & $\begin{array}{r}7 \\
1.93 \\
29.17 \\
11.29 \\
\end{array}$ & $\begin{array}{r}1 \\
0.28 \\
4.17 \\
385 \\
\end{array}$ & $\begin{array}{r}7 \\
1.83 \\
29.17 \\
14.89 \\
\end{array}$ & $\begin{array}{r}0 \\
0.00 \\
0.00 \\
0.00\end{array}$ & $\begin{array}{r}4 \\
1.10 \\
16.67 \\
23.53 \\
\end{array}$ & $\begin{array}{r}0 \\
0.00 \\
0.00 \\
0.00\end{array}$ & $\begin{array}{r}24 \\
6.61\end{array}$ \\
\hline TOTAL & $\begin{array}{r}1 \\
0.28\end{array}$ & $\begin{array}{r}1.16 \\
31.96\end{array}$ & $\begin{array}{r}78 \\
21.49\end{array}$ & $\begin{array}{r}62 \\
17.08\end{array}$ & $\begin{array}{r}26 \\
7.16\end{array}$ & $\begin{array}{r}47 \\
12.95\end{array}$ & $\begin{array}{r}14 \\
386\end{array}$ & $\begin{array}{r}17 \\
.4 .68\end{array}$ & $\begin{array}{r}2 \\
0.55\end{array}$ & $\begin{array}{r}363 \\
100.00\end{array}$ \\
\hline
\end{tabular}




\section{COMPLICACIONES PERINATALES VS. DIAGNOSTICO EGRESO \\ EN EL TRATAMIENTO ACTUAL}

(B)

\begin{tabular}{|c|c|c|c|c|c|c|c|c|c|c|}
\hline & SIN DATO & P. E. & I. E. & E. & EVHC + P.E & EVHC + I.E. & EVHC + E & E.V. H.C. & OTRO & TOTAL \\
\hline $\begin{array}{l}\text { FRECUENGIA } \\
\% \\
\% \times \text { FILA } \\
\% \times \text { COLUMNA }\end{array}$ & $\begin{array}{l}0 \\
\dot{.} \\
\dot{.}\end{array}$ & $\begin{array}{r}0 \\
0.00 \\
0.00 \\
0.00 \\
\end{array}$ & $\begin{array}{r}0 \\
0.00 \\
0.00 \\
0.00\end{array}$ & $\begin{array}{r}4 \\
1.02 \\
80.00 \\
5.26 \\
\end{array}$ & $\begin{array}{r}0 \\
0.00 \\
0.00 \\
0.00 \\
\end{array}$ & $\begin{array}{r}1 \\
0.20 \\
20.00 \\
227 \\
\end{array}$ & $\begin{array}{r}0 \\
0.00 \\
0.00 \\
0.00 \\
\end{array}$ & $\begin{array}{r}0 \\
0.00 \\
0.00 \\
0.00 \\
\end{array}$ & $\begin{array}{r}0 \\
0.00 \\
0.00 \\
0.00 \\
\end{array}$ & $\begin{array}{r}5 \\
1.28\end{array}$ \\
\hline SIN COMPLICACION & $\begin{array}{l}0 \\
. \\
. \\
\end{array}$ & $\begin{array}{r}115 \\
29.41 \\
34.85 \\
94.26 \\
\end{array}$ & $\begin{array}{r}48 \\
12.28 \\
14.55 \\
88.89 \\
\end{array}$ & $\begin{array}{r}57 \\
14.58 \\
17.27 \\
75.00 \\
\end{array}$ & $\begin{array}{r}50 \\
12.79 \\
15.15 \\
83.33 \\
\end{array}$ & $\begin{array}{r}36 \\
9.21 \\
10.91 \\
81.82 \\
\end{array}$ & $\begin{array}{r}10 \\
256 \\
3.03 \\
50.00 \\
\end{array}$ & $\begin{array}{r}11 \\
2.81 \\
3.33 \\
100.00 \\
\end{array}$ & $\begin{array}{r}3 \\
0.77 \\
0.91 \\
75.00 \\
\end{array}$ & $\begin{array}{r}330 \\
84.40\end{array}$ \\
\hline $\begin{array}{l}\text { MUERTE } \\
\text { EXTRAHOSPITALARIA }\end{array}$ & $\begin{array}{l}0 \\
. \\
. \\
.\end{array}$ & $\begin{array}{r}3 \\
0.77 \\
14.29 \\
2.48 \\
\end{array}$ & $\begin{array}{r}1 \\
0.26 \\
4.76 \\
1.85\end{array}$ & $\begin{array}{r}5 \\
1.28 \\
23.81 \\
6.58\end{array}$ & $\begin{array}{r}1.02 \\
19.05 \\
6.67\end{array}$ & $\begin{array}{r}1.02 \\
19.05 \\
9.09\end{array}$ & $\begin{array}{r}1.02 \\
19.05 \\
20.00\end{array}$ & $\begin{array}{l}0 \\
0.00 \\
0.00 \\
0.00\end{array}$ & $\begin{array}{l}0 \\
0.00 \\
0.00 \\
0.00\end{array}$ & $\begin{array}{r}21 \\
5.37\end{array}$ \\
\hline $\begin{array}{l}\text { MUERTE } \\
\text { INTRAHOSPITALARIA } \\
\text { ANTEPARTO }\end{array}$ & $\begin{array}{l}0 \\
\cdot \\
. \\
.\end{array}$ & $\begin{array}{r}3 \\
0.77 \\
18.75 \\
2.46 \\
\end{array}$ & $\begin{array}{r}2 \\
0.51 \\
12.50 \\
3.70 \\
\end{array}$ & $\begin{array}{r}1.28 \\
31.25 \\
6.58 \\
\end{array}$ & $\begin{array}{r}1 \\
0.26 \\
6.25 \\
1.67 \\
\end{array}$ & $\begin{array}{r}2 \\
0.51 \\
12.50 \\
4.55 \\
\end{array}$ & $\begin{array}{r}2 \\
0.51 \\
12.50 \\
10.00 \\
\end{array}$ & $\begin{array}{l}0 \\
0.00 \\
0.00 \\
0.00 \\
\end{array}$ & $\begin{array}{r}1 \\
0.26 \\
6.25 \\
25.00 \\
\end{array}$ & $\begin{array}{r}16 \\
4.09\end{array}$ \\
\hline $\begin{array}{l}\text { MUERTE } \\
\text { INTRAHOSPITALARIA } \\
\text { INTRAPARTO }\end{array}$ & $\begin{array}{l}0 \\
\dot{.} \\
. \\
\end{array}$ & $\begin{array}{r}0 \\
0.00 \\
0.00 \\
0.00 \\
\end{array}$ & $\begin{array}{r}1 \\
0.26 \\
33.33 \\
1.85 \\
\end{array}$ & $\begin{array}{r}1 \\
0.26 \\
33.33 \\
1.32 \\
\end{array}$ & $\begin{array}{r}1 \\
0.26 \\
33.33 \\
1.67 \\
\end{array}$ & $\begin{array}{l}0 \\
0.00 \\
0.00 \\
0.00 \\
\end{array}$ & $\begin{array}{l}0 \\
0.00 \\
0.00 \\
0.00 \\
\end{array}$ & $\begin{array}{l}0 \\
0.00 \\
0.00 \\
0.00 \\
\end{array}$ & $\begin{array}{l}0 \\
0.00 \\
0.00 \\
0.00 \\
\end{array}$ & $0.7 \frac{3}{7}$ \\
\hline $\begin{array}{l}\text { MUERTE } \\
\text { NEONATAL }\end{array}$ & $\begin{array}{l}0 \\
\text {. }\end{array}$ & $\begin{array}{r}1 \\
0.26 \\
6.25 \\
0.82 \\
\end{array}$ & $\begin{array}{r}2 \\
0.51 \\
12.50 \\
3.70 \\
\end{array}$ & $\begin{array}{r}4 \\
1.02 \\
25.00 \\
5.26 \\
\end{array}$ & $\begin{array}{r}4 \\
1.02 \\
25.00 \\
6.67 \\
\end{array}$ & $\begin{array}{r}1 \\
0.26 \\
6.25 \\
2.27 \\
\end{array}$ & $\begin{array}{r}4 \\
1.02 \\
25.00 \\
20.00 \\
\end{array}$ & $\begin{array}{l}0 \\
0.00 \\
0.00 \\
0.00\end{array}$ & $\begin{array}{r}0 \\
0.00 \\
0.00 \\
0.00\end{array}$ & $\begin{array}{r}16 \\
4.09\end{array}$ \\
\hline TOTAL & $\dot{.}$ & $\begin{array}{r}122 \\
31.20\end{array}$ & $\begin{array}{r}54 \\
13.81\end{array}$ & $\begin{array}{r}76 \\
19.44 \\
\end{array}$ & $\begin{array}{r}60 \\
15.35 \\
\end{array}$ & $\begin{array}{r}44 \\
11.25\end{array}$ & $\begin{array}{r}20 \\
5.12 \\
\end{array}$ & $\begin{array}{r}11 \\
2.81\end{array}$ & 1.02 & $\begin{array}{r}391 \\
100.00\end{array}$ \\
\hline
\end{tabular}

\section{MORTALIDAD FETAL Y NEONATAL INTRAHOSPITALARIA SEGUN EL DIAGNOSTICO}

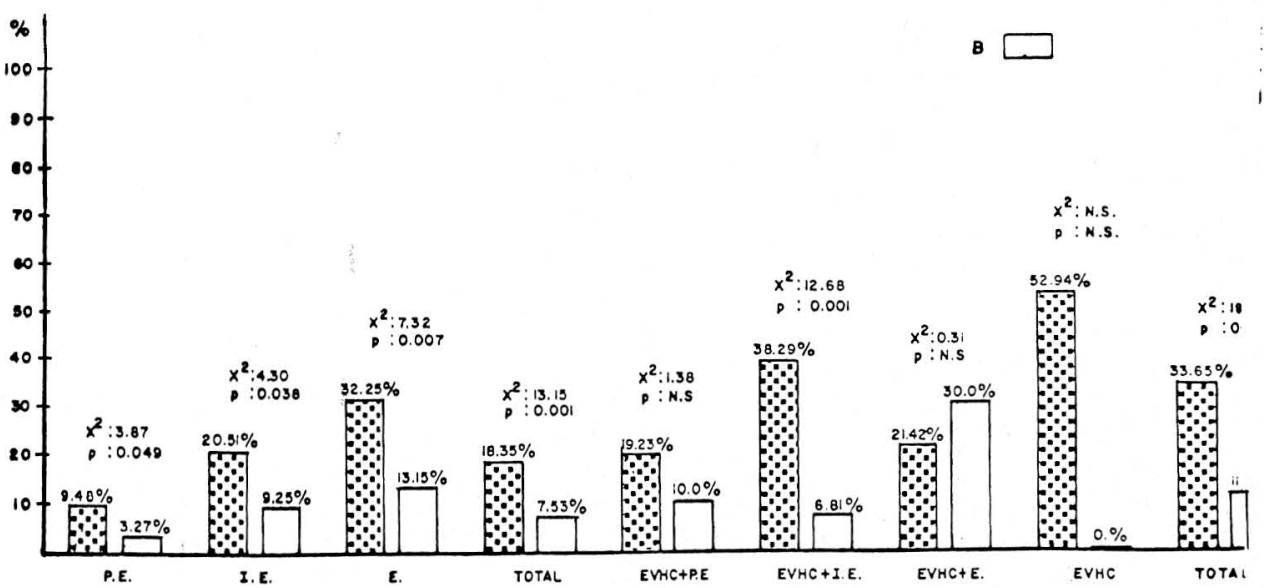




\section{COMENTARIOS}

Los dos grupos de pacientes analizados pueden considerarse similares por cuanto no hay diferencia significativa. Los resultados, por consiguiente, pueden considerarse adecuados.

Un hecho notorio es la brusca disminución de la mortalidad materna, influye en esto la motivación que se efectuó a nivel del personal asistencial de los centros periféricos, la hospitalización más temprana de las pacientes. En el grupo A existe una mayor mortalidad, pero es necesario considerar que la mayoría de las pacientes fallecidas ingresaron en un período final en el cual todos los esfuerzos médicos para recuperar las condiciones vitales ya fueron definitivamente infructuosos.

En el segundo grupo ingresó un menor número de pacientes insalvables y la recuperación de sus condiciones vitales fue más oportuna. Por otra parte en el grupo $\mathrm{B}$ el tipo de tratamiento fue más.agresivo y por la conducta obstetrica más intervencionista que en el primer grupo analizado.

De esta manera, la eclampsia que durante el primer período analizado fue la primera causa de mortalidad materna en el Instituto, pasa a ser la última en el segundo período. Demostración clara de como la iniciación temprana del tratamiento, la hospitalización precoz $\mathrm{y}$ las conductas intervencionistas pueden mejorar el pronóstico de la entidad.

No se apreció mortalidad materna en las pacientes con hipertensión inducida por el embarazo, en las pacientes del grupo $B$, lo que contrasta con las cifras de mortalidad relatadas por Jubiz (7) establecidas en el $2.3 \%$.

La mortalidad materna del grupo B, 9 por cien mil pacientes, relacionada con el número de partos en el Instituto $\mathrm{Ma}$ terno Infantil de Bogotá es igual a la relatada para Inglaterra y Gales en donde la mortalidad por toxemia es de 8.9 casos por cien mil nacimientos $\mathrm{y}$ esta cifra relatada por Deborah Maine, M.P. $H$. del Centro de Población y Salud Familiar, Columbia University es comentada como un gran alcance logrado sobre la disminución de la mortalidad materna debido a la planificación familiar, (8).

La mortalidad perinatal global en los estados hipertensivos implica una mayor dificultad para lograr una reducción notoria. La labilidad fetal es mayor que la materna. A pesâr de esto se han logrado avances significativamente prometedores, se ha reducido de un $26.16 \%$ al $14.32 \%$, cifra ésta igual a la reportada por Tchobroutsky et. al. en la mortalidad de Post-Royal Paris, 1982 (10). Esta disminución de la mortalidad perinatal en las. enfermedades hipertensivas, agudas y crónicas se hace más notoria si se analiza la mortalidad perinatal intrahospitalaria en donde si se puede apreciar claramente la acción que el tratamiento y las conductas adoptadas ejercen sobre el pronóstico del producto; en este grupo la mortalidad se reduce de un $22.83 \%$ al 8.95 .

La mortalidad perinatal en la preeclampsia descrita por Jubiz (7) del $8.27 \%$ es similar a la mortalidad de las pacientes del grupo $\mathrm{B}, 7.53 \%$.

Comparadas la mortalidad perinatal en las pacientes del grupo B con las cifras presentadas por Hellman y Pritchard (9), se obtiene los siguientes datos: Mortalidad en la preeclampsia: Grupo $\mathrm{B}=5.1 \%$, Hellman $=5.7 \%$ En la eclampsia: Grupo $\mathrm{B}=13.15 \%$, Hellman $=1 \% .7 \%$. En la enfermedad hipertensiva crónica con preeclampsia sobreagregada en el grupo $\mathrm{B}=8.65 \%$, Hellman $=24.6 \%$. No se presenta estadística en enfermedad hipertensiva crónica con eclampsia sobreagregada.

Los datos son concluyentes, se ha avanzado notoriamente en el dominio de 
las consecuencias de la enfermedad hipertensiva en nuestro medio.

De esta situación puede inferirse que los análisis fetales antenatales,.ecografía, tococardiografía y los estudios bioquímicos del líquido amniótico influyen muy positivamente en la reterminación de la fecha en que se hace necesario interrumpir el embarazo y en la escogencia de la vía por la cual debe efectuarse la extracción fetal.

Un segundo factor ha influido en la reducción de la mortalidad del recién nacido, es la oportuna e idónea atención del niño recién nacido durante las primeras horas de vida, aunque es de notar que en nuestro servicio todavía existe deficiencia en la atención continuada del recién nacido durante los primeros días. Estos niños requieren una atención intensiva para lograr que la supervivencia sea mayor. Esta deficiencia ha sido la causa por la cual la disminución de la mortalidad neonatal, a pesar de ser significativa, no lo sea tan notoria como la disminución de la mortalidad en el período antenatal e intraparto.

La mortalidad perinatal está correlacionada con la edad gestacional y el peso del recién nacido, siendo mayor cuanto menor sea el peso fetal. Sin embargo, la mortalidad se ve reducida en el segundo grupo de pacientes especialmente en los niños que tienen un peso comprendido entre 1.000 y 2.500 grs. No hay diferencia entre los niños de mayor peso.

Las nuevas medidas adoptadas en la sección de prematuros, consistentes en la entrega del prematuro a la madre, no importa cual sea el peso, siempre y cuando sus condiciones vitales se encuentren .normales, también ha favorecido el pronóstico del recién nacido, se han disminuido de esta manera los problemas infecciosos inherentes a las salas cunas. Sin embargo, los niños nacidos en condiciones de grave retraso de crecimiento y con deterioro en sus funciones respiratorias y metabólicas requieren algo más que el seno materno lo cual aún no disponemos en nuestro hospitál.

En relación al tipo de parto, la mortalidad es similar en. los partos atendidos por vía vaginal $y$ los intervenidos por cesárea en las pacientes del grupo B. De ello se ciesprende que cuando la vía del parto es escogida adecuadamente no hay influencia sobre el pronóstico del recién nacido.

Comparada la mortalidad de los dos grupos si hay una deficiencia apreciable y significativa. En primer lugar, la conducta fue más espectante $y$ conservadora en el grupo $A$, en cambio fue más activa selectiva y quirúrgica en las pacientes del grupo B. De hecho, se han efectuado un número mayor de cesáreas perc esto ha disminuido la frecuencia del sufrimiente fetal intraparto sobreagregado al sufrimiento crónico existente en lós estados avanzados de la entidad.

La mortalidad perinatal en las pacientes intervenidas por cesárea en el grupo A fue de un $21.9 \%$ en cambio en el grupo B fue de un $9.28 \%$ lo que indica que la cesárea en el primer grupo se indico tardíamente.

Por otra parte, la mortalidad en las pacientes atendidas por vía vaginal en el grupo A fue del $22.64 \%$ en cambio en el grupo B la mortalidad es del $8.83 \%$. Nuevamente, la monitoría fetal favorece estos resultados. La prueba de Pose nos permite analizar la factibilidad del parto vaginal.

La mortalidad perinatal se correlaciona intimamente con el estado evolutivo de la enfermedad; en ambos grupos se aprecia que la mortalidad perinatal progresa, desde la preeclampsia hasta la eclampsia y desde la enfermedad vascular hipertensiva sola hasta la enfermedad vascular hipertensiva con eclampsia sobreagregada. 
La mortalidad perinatal es específicamente alta en las pacientes con E.V.H.C. con preeclampsia o eclampsia sobreagregada. Si consideramos que estas pérdidas se presentarán, muy seguramente, en embarazos sucesivos, es recomendable y sano concientizar a las pacientes para la práctica de métodos anticonceptivos de carácter definitivo cuando sean grandes multíparas.

Es de anotar que aun es escandalosa la cifra de pacientes que ingresa en estado de eclampsia o con complicaciones post-eclámpticas; mientras exista la mortalidad tanto materna como fetal continuará siendo también alarmante.

\section{CONCLUSIONES}

Si actualmente no ha sido identificado plenamente el factor etiológico de la hipertensión inducida por el embarazo, tanto en pacientes normotensas previamente como en pacientes con hipertensión crónica antecediendo al embarazo, si se conoce que la hipertensión es la causante de las lesiones arteriolares de todos los órganos expuestos y conlleva el riesgo de convulsiones, hemorragia cerebral, insuficiencia ventricular izquierda, alteraciones renales y coagulación intravascular.

Para el obstetra, en su práctica habitual, es imperativo tener en cuenta la rapidez con que aparece la lesión arterial. Los vasodilatadores aplicados oportunamente evitan esta lesión causal del grave peligro de muerte. Los otros signos coexistentes, intimamente relacionados a la hipertensión son secundarios a la misma y por tanto al vencer el vasoespasmo desaparecen los otros síntomas.

Inicialmente el tratamiento de los estados hipertensivos se hizo con la apli cación continua y prolongada de diuréticos y barbitúricos. Los primeros administrados con la esperanza de que al disminuir la concentración sódica del plasma la hipertensión cediera, siguiendo las pautas de tratamiento de la hipertension esencial en pacientes no' embarazadas. Se desconocía por otra parte que la hipertensión aguda del embarazo está acompañada de hipovolemia. Los resultados de este tratamiento fueron casi de tipo cosmético ya que desaparecía el signo edema rápidamente pero la función renal y el flujo placentario disminuían más, comprometiendo mas profundamente las lesiones $y$ daño fetal $y$ renal.

Los barbitúricos formulados a largo plazo fueron indicados con 'a intención de sedar el sistema nervioso central al cual se responsabilizaba, en parte, la hipertensión arterial. Hoy se concee que las crisis emotivas pueden ser responsables de crisis hipertensivas ocasionales pero no causales de la hipertensión de base. Los barbitúricos ordenados por largo tiempo inhiben la maduración pulmonar y los movimientos respiratorios fetales intrauterinos que desarrollan la musculatura que hace posible una buena adaptación al nuevo tipo de respiración del recién nacido.

Un método bastante sencillo para curar la enfermedad hipertensiva aguda del embarazo es la terminación del mismo. Esta opciórt debe analizarse con sumo cuidado teniendo en cuenta el riesgo de la paciente $y$ el riesgo del feto.

Desde el punto de vista materno, la continuación del embarazo puede permitirse si las lesiones existentes son mínimas lo cual se encuentra solamente cuando la entidad ha sido descubierta tempranamente y el tratamiento ejecutado a plenitud en todos sus conceptos. En otras situaciones, las lesiones arteriolares ya están establecidas y la prolongación del embarazo repercutirá inevitablemente en un desenlace fatal.

Desde el punto de vista fetal caben las mismas consideraciones en relación al 
tiempo en que se diagnostica y trata la enfermedad.

Para valorar el compromiso fetal es necesario disponer de elementos de trabajo perfeccionados tales como la ecografía, cardiotocografía obstétrica, métodos de titulación de elementos del líquido amniótico y aún valoraciones hormonales por radioinmunoanálisis. Estos elementos solo pueden hallarse en Institutos de nivel III de atención y por tanto todas estas pacientes deben ser tratadas allí.

La comparación de los dos tipos de tratamiento efectuados en el Instituto Materno Infantil en dos etapas diferentes ha demostrado que la suspensión de los diuréticos, los barbitúricos y la introducción del tratamiento rutinario a base de hipotensores, ha repercutido en las cifras de mortalidad tanto materna como fetal.

Sin embargo, es necesario tener presente que no solamente este factor ha sido el responsable de esta disminución; la adquisición de un ecógrafo $y$ de un monitor para la Sección de Toxemias ha sido de una invaluable ayuda para determinar el grado de compromiso fetal y la fecha en la cual es necesario desembarazar a la paciente y escoger la vía por la cual esto sea posible con el menor riesgo. En el grupo $B$ de pacientes se ha logrado una disminución de la mortalidad fetal, especialmente en el grupo de niños prematuros $y$ en la disminución de la mortalidad ante e intraparto.

Es preciso reconocer que el manejo inmediato del recién nacido fue más riguroso $y$ dedicado en el grupo $B$ de pacientes que en el $A$, nuevas pautas para la rehabilitación del recién nacido, recientemente instituidas en el servicio, han permitido una mayor recuperación de niños gravemente afectados por la hipoxia.
El uso de vasodilatadores por vía parenteral repercute especialmente en la reducción de la mortalidad materna previniendo graves alteraciones hemorrágicas en pacientes con hipertensión maligna.

Por lo expuesto y por los resultados obtenidos en la hipertensión del embarazo es una necesidad urgente disminuir la tensión arterial por medio de hipotensores $\mathrm{y}$ vasodilatadores $\mathrm{y}$ suspender totalmente la administración de diuréticos salvo los casos en los cuales se haga indispensable su uso: I.C.C., edema pulmonar, hipertensión resistente a los vasodilatadores específicos $y$ en los casos en los cuales se utilicen como prueba de valoración de función renal.

Cuando la eclampsia se presenta en el período anteparto, el pronóstico de madre e hijo empeoran con el tiempo que se retarde la expulsión o extracción fetal. Esto se ha tenido en cuenta en el grupo $B$ de pacientes, en el grupo A era frecuente prolongar el período del embarazo con el objeto de obtener un mayor crecimiento y maduración fetal. En el análisis no se hizo una evaluación precisa de esta condición que deberá estudiarse cuidadosamente bajo otros parámetros diferentes a los usados aquí.

Una mayor vigilancia a nivel prenatal se hace urgente en nuestro medio, la morbimortalidad depende de la precocidad con que se descubra la enfermedad, la aplicación oportuna de los fármacos y la adopción esmerada de una conducta seleccionada exhaustivamente.

Aunque las cifras de mortalidad en el grupo B sean bajas, estas pueden volver a ascender ya que en la periferia las pacientes aun no asisten regularmente a la consulta prenatal y lo que es peor aún, en algunas consultas prenatales oficiales este control es desesperadamente defastuoso. 


\section{RESUMEN}

En el grupo A se trataron 363 pacientes de las cuales el $31.96 \%$ fueron preeclámpticas, $21.49 \%$ presentaron preeclampsia grave, inminencia de eclampsia $17.08 \%$ tuvieron eclampsia, $7.16 \%$ de pacientes presentaron E.V.H.C. con preeclampsia sobreagregada, $12.95 \%$ tuvieron E.V.H.C. con inminencia de eclampsia, $3.86 \%$ presentaron E.V.H.C. con eclampsia sobreagregada y $4.68 \%$ fueron E.V.H.C. puras.

La mortalidad materna en las pacientes hipertensas, en el grupo $A$ fue el $5.23 \%$ y en el grupo B fue el $0.26 \%$.

Se analizaron dos grupos de pacientes sometidas a dos tipos de tratamientos en los estados hipertensivos del embarazo. El primer grupo A tratado sistemáticamente con diuréticos, barbitúricos a largo plazo y conductas espectantes para lograr mayor maduración fetal. El seguindo grupo B tratado especialmente con vasodilatadores en el cual las conductas, apoyadas en una evaluación fetal más rigurosa, fue más activa e intervancionista.
En relación con el número de partos atendidos en el Instituto, la mortalidad materna en el grupo $A$ es de 13 por 10.000 partos $y$ en el grupo $B$ de 0.9 por 10.000 partos.

La mortalidad por toxemia representa el $41 \%$ de la mortalidad materna total del Instituto en el grupo $A$ de pacientes analizadas y el $4.3 \%$ en el grupo $B$ de pacientes.

La mortalidad perinatal, se desglosa minuciosamente; en general la mortalidad perinatal total del grupo $A$ es del $26.17 \%$ v en el grupo $B$ es del $14.32 \%$.

La mortalidad perinatal tiene una diferencia altamente significativa por los análisis estadísticos demostrándose que el segundo grupo de pacientes se han beneficiado por el nuevo sistema de tratamiento.

Los beneficios obtenidos no pueden imputarse a un solo parámetro de tratamiento, éstos son el resultado de la suma de todas las nuevas pautas establecidas.

\section{MATERNAL AND PERINATAL MORTALITY IN HYPERTENSIVE GESTATIONS}

\section{SUMMARY}

This study analized two groups of patients treated for hypertension during pregnancy with two different methods. Group A was systematically treated with diuretic medicines, barbiturates and adequate expectation as a means of obtaining more fetal maturity. Group B was treated mainly with vessel dilators and a more active intervention supported by a more careful fetal assessment.

Group A involved 363 patients, 31 . $96 \%$ of which had pre-eclampsia, 21. $49 \%$ had severe pre-eclampsia, $17.08 \%$ had eclampsia $7.16 \%$ reported E.V.H.C. with aggregate pre-eclampsia, 12.95 had
E.V.H.C. with impending eclampsia, 3.86 had E.V.H.C. with aggregate eclampsia and 4.68 were only E.V.H.C.

In group A maternal mortality among hypertensive women was 5.23 and in Group B it was $0.26 \%$.

In relation to the number of deliveries at the Institute, maternal mortality in group $A$ is 13 for every 10.000 deliveries, and in Group B it is 0.9 for every 10.000 deliveries.

Mortality derived from toxemia represents $41 \%$ of the global maternal mortality at the Institute in Group A, and $4.3 \%$ in Group B. 
Perinatal mortality is analyzed in detail in this study. Global perinatal mortality in Group $A$ is $26.17 \%$ and in Group B it is $14.32 \%$.

Perinatal mortality presents a highly significant difference as shown by, the statistical analyses, thus confirming the

\section{BIBLIOGRAFIA}

1. CAMPBELL, D.M., MAcGILLIVRAY, I. The effect of low calorie diet or a diuretic on the incidence of pre eclampsia and birth weight. British Journal of Obstetrics and Gynecology, 82, 5721975.

2. MACGILLIVRAY, I., Equilibrio de sodio y agua. Ginecología y Obstetricia. Temas actuales. 549, diciembre, 1977.

3. SANCHEZ TORRES, F., GUEVARA, J. H., GOMEZ PALACINO, J.A. Eclampsia anteparto. Rev. Col. de Obst. y Gine. 225, XVI, 1965.

4. SANCHEZ TORRES, F., Eclamspia intraparto. Rev. Col. Obst. y Ginec. 397, XV. 1974.

5. ROJAS R., L., Mortalidad materna en el Instituto Materno Infantil de Bogotá. Rev. Col. Obst. Y Ginec. 395, XXV. 1974. fact that the second group of patients benefits from the new methods of treatment.

The benefits, however, cannot be attributed only to one of the elements of the treatment; they are the result of all the new treatment criteria combined.

6. RODRIGUEZ S.A., PEDRAZA G., J., GUTIERREZ E., Mortalidad perinatal en Instituciones Hospitalarias de Bogotá. Rev. Col. de Obst. y Ginec. 273, XXVII, 1976.

7. JUBIZ M.A., Morbimortalidad materna y perinatal en pacientes con preeclampsia y eclampsia. Rev. Col. de Obst. y Ginec. 294 XXXI.

8. MAINE, D., Planificación Familiar: Su efecto en la salud de la mujer y el niño. Center for Population and Family Health. Faculty of Medicine. Columbia University. 1981 N.Y.

9 HELLMAN L.M., WILLIAMS Obstetricia Salvat Editores 1973.

10. TCHOBROUTSKY C., DUMEZ Y., LIRZIND, HORNYCH H., AMIELTTISON C. Hipertensión arterial durante el embarazs La Nouvelle Presse Medicale. 637, 11-12: 1982. 